\title{
Integral Cayley graphs over abelian groups
}

\author{
Walter Klotz and Torsten Sander \\ Institut für Mathematik \\ Technische Universität Clausthal, Germany \\ klotz@math.tu-clausthal.de \\ torsten.sander@math.tu-clausthal.de
}

Submitted: Dec 8, 2009; Accepted: May 20, 2010; Published: May 25, 2010

Mathematics Subject Classification: 05C25, 05C50

\begin{abstract}
Let $\Gamma$ be a finite, additive group, $S \subseteq \Gamma, 0 \notin S,-S=\{-s: s \in S\}=S$. The undirected Cayley graph $\operatorname{Cay}(\Gamma, S)$ has vertex set $\Gamma$ and edge set $\{\{a, b\}: a, b \in \Gamma$, $a-b \in S\}$. A graph is called integral, if all of its eigenvalues are integers. For an abelian group $\Gamma$ we show that $\operatorname{Cay}(\Gamma, S)$ is integral, if $S$ belongs to the Boolean algebra $B(\Gamma)$ generated by the subgroups of $\Gamma$. The converse is proven for cyclic groups. A finite group $\Gamma$ is called Cayley integral, if every undirected Cayley graph over $\Gamma$ is integral. We determine all abelian Cayley integral groups.
\end{abstract}

\section{Introduction}

Eigenvalues of an undirected graph $G$ are the eigenvalues of an arbitrary adjacency matrix of $G$. Harary and Schwenk [9] defined $G$ to be integral, if all of its eigenvalues are integers. Since then many integral graphs have been discovered, for a survey see [4]. Nevertheless, as is shown in [2], the probability of a labeled graph on $n$ vertices to be integral is at most $2^{-n / 400}$ for sufficiently large $n$. Known characterizations of integral graphs are restricted to certain graph classes. Here we proceed towards a characterization of integral Cayley graphs over abelian groups.

Let $\Gamma$ be a finite, additive group, $S \subseteq \Gamma, 0 \notin S,-S=\{-s: s \in S\}=S$. The undirected Cayley graph $\operatorname{Cay}(\Gamma, S)$ has vertex set $\Gamma$. Vertices $a, b \in \Gamma$ are adjacent if $a-b \in S$. For general properties of Cayley graphs we refer to Godsil and Royle [8] or Biggs [5]. Abdollahi and Vatandoost [1] show that there are exactly seven connected cubic integral Cayley graphs. So [15] presents a characterization of integral circulant graphs, which are Cayley graphs over cyclic groups. In this paper we prove for an abelian group $\Gamma$ that $\operatorname{Cay}(\Gamma, S)$ is integral, if $S$ belongs to the Boolean algebra $B(\Gamma)$ generated by the subgroups of $\Gamma$. By the result of So the converse turns out to be true for cyclic groups. We conjecture it to be true for abelian groups in general. 
A finite group $\Gamma$ is called Cayley integral, if every undirected Cayley graph over $\Gamma$ is integral. We show that all nontrivial abelian Cayley integral groups are represented by

$$
Z_{2}^{n}, Z_{3}^{n}, Z_{4}^{n}, Z_{2}^{m} \otimes Z_{3}^{n}, Z_{2}^{m} \otimes Z_{4}^{n}, \quad m \geqslant 1, n \geqslant 1
$$

Here $Z_{k}=\{0,1, \ldots, k-1\}$ denotes the (additive) cyclic group of integers modulo $k$.

The Hamming graph $\operatorname{Ham}\left(m_{1}, \ldots, m_{r} ; D\right)$ has vertex set $Z_{m_{1}} \otimes \cdots \otimes Z_{m_{r}}$. Vertices $x \neq y$ are adjacent, if their Hamming distance is in a list $D$ of possible distances. All Hamming graphs are proven to be integral Cayley graphs, which extends a partial result in [14]. Moreover, we show that certain graphs associated with the Sudoku puzzle and with pandiagonal Latin squares are integral Cayley graphs.

We remark that every set $S$ in the Boolean algebra $B(\Gamma)$ satisfies $S=-S$. For the construction of a Cayley graph $\operatorname{Cay}(\Gamma, S)$ we use only those $S \in B(\Gamma)$ which do not contain the additive identity 0 of $\Gamma$.

\section{Integral subsets}

Let $Z$ be the set of all integers, $M$ a finite, nonempty set, and $f$ a complex valued function on $M, f: M \rightarrow \mathbb{C}$. A subset $A \subseteq M$ is called $f$-integral, if

$$
f(A)=\sum_{a \in A} f(a) \in Z .
$$

We agree upon $f(\emptyset)=0$. So the empty set is always $f$-integral. The complement of $A \subseteq M$ is $\bar{A}=M \backslash A$. The following simple Lemma is due to $f(\bar{A})=f(M)-f(A)$.

Lemma 1. Let $M$ be $f$-integral and $A \subseteq M$. Then $A$ is $f$-integral, if and only if $\bar{A}$ is $f$-integral.

A family $\Omega=\left\{A_{1}, \ldots, A_{n}\right\}$ of subsets of $M$ is called intersection stable, if $A_{i} \cap A_{j} \in \Omega$ for every $i, j \in\{1, \ldots, n\}$.

Lemma 2. Let $\Omega=\left\{A_{1}, \ldots, A_{n}\right\}$ be an intersection stable family of $f$-integral subsets of $M$. If $M$ is $f$-integral, then:

1. $\bar{A}_{i} \cap A_{j}$ is $f$-integral for every $i, j \in\{1, \ldots, n\}$.

2. $A_{i_{0}} \cap \bar{A}_{i_{1}} \cap \ldots \cap \bar{A}_{i_{k}}$ is $f$-integral for every $k \geqslant 1$ and $\left\{i_{0}, i_{1}, \ldots, i_{k}\right\} \subseteq\{1, \ldots, n\}$. This remains true, if $A_{i_{0}}$ is missing, respectively $A_{i_{0}}=M$.

Proof. 1. For $i \neq j$ the set $A_{i} \cap A_{j} \in \Omega$ is $f$-integral and so

$$
f\left(\bar{A}_{i} \cap A_{j}\right)=f\left(A_{j}\right)-f\left(A_{i} \cap A_{j}\right) \in Z .
$$

2. Without loss of generality let $i_{j}=j$ for $j=0, \ldots, k, A_{0} \in \Omega$ or $A_{0}=M$,

$$
A=A_{0} \cap \overline{A_{1}} \cap \ldots \cap \bar{A}_{k} .
$$


In view of Lemma 1 it is sufficient to show that $\bar{A}$ is $f$-integral. By de Morgan's rule we have

$$
\bar{A}=\bar{A}_{0} \cup A_{1} \cup \ldots \cup A_{k} .
$$

We apply the principle of inclusion and exclusion (see [11]) to determine $f(\bar{A})$.

$$
\begin{gathered}
f(\bar{A})=\sum_{p=1}^{k+1}(-1)^{p-1} s_{p}, \\
s_{p}=\sum_{0 \leqslant j_{1}<\ldots<j_{p} \leqslant k} f\left(B_{j_{1}} \cap \ldots \cap B_{j_{p}}\right),
\end{gathered}
$$

where $B_{0}=\bar{A}_{0}$ and $B_{j}=A_{j}$ for every $j \in\{1, \ldots, k\}$. We show that all terms in the sum (2) represent integers. For $j_{1}>0$ we have

$$
f\left(B_{j_{1}} \cap \ldots \cap B_{j_{p}}\right)=f\left(A_{j_{1}} \cap \ldots \cap A_{j_{p}}\right) \in Z
$$

because $A_{j_{1}} \cap \ldots \cap A_{j_{p}} \in \Omega$ is $f$-integral. If $j_{1}=0$ then $B_{j_{1}} \cap \ldots \cap B_{j_{p}}$ reduces to

$$
\bar{A}_{0} \cap A_{j_{2}} \cap \ldots \cap A_{j_{p}}=\bar{A}_{0} \cap A_{j} \text { for some } A_{j} \in \Omega
$$

because $\Omega$ is intersection stable. If $A_{0}=M$ then $f\left(\bar{A}_{0} \cap A_{j}\right)=f(\emptyset)=0$. If $A_{0} \in \Omega$ then $f\left(\bar{A}_{0} \cap A_{j}\right) \in Z$ follows from part 1 of this lemma.

Let $A_{1}, \ldots, A_{n}$ be subsets of $M$. We denote the Boolean algebra generated by $A_{1}, \ldots$, $A_{n}$ in $M$ by $B\left(A_{1}, \ldots, A_{n} ; M\right)$. It is the smallest system of subsets of $M$ that contains $A_{1}, \ldots, A_{n}$ and is invariant under the set operations union, intersection, and forming the complement. It is well known (see e.g Cohn [6]) that $B\left(A_{1}, \ldots, A_{n} ; M\right)$ consists exactly of those sets $A \subseteq M$ which can be represented in disjunctive normal form by $A_{1}, \ldots, A_{n}$ :

$$
\begin{aligned}
& A=\bigcup_{j=1}^{k} D_{j}, D_{j}=\bigcap_{l=1}^{n_{j}} W_{j, l}, \\
& W_{j, l} \in\left\{A_{1}, \ldots, A_{n}, \overline{A_{1}}, \ldots, \overline{A_{n}}\right\} \text { for every } j, l .
\end{aligned}
$$

If the set system $\Omega=\left\{A_{1}, \ldots, A_{n}\right\}$ is intersection stable, then the sets $D_{j}$ in (3) can be reduced to

$$
D_{j}=A_{j, 0} \cap \bar{A}_{j, 1} \cap \ldots \cap \bar{A}_{j, m_{j}},
$$

where every $A_{j, l} \in \Omega$. We may have $m_{j}=0$ and the term $A_{j, 0}$ may be missing.

Lemma 3. Let $\Omega=\left\{A_{1}, \ldots, A_{n}\right\}$ be an intersection stable family of subsets of $M$. If $M$ and all sets $A_{1}, \ldots, A_{n}$ are $f$-integral, then every set $A \in B\left(A_{1}, \ldots, A_{n} ; M\right)$ is also $f$-integral. 
Proof. Every set $A \in B\left(A_{1}, \ldots, A_{n} ; M\right)$ can be written in disjunctive normal form according to (3). Once more we apply the inclusion-exclusion principle, this time to determine $f(A)$.

$$
\begin{gathered}
f(A)=\sum_{p=1}^{k}(-1)^{p-1} s_{p} \\
s_{p}=\sum_{1 \leqslant i_{1}<\ldots<i_{p} \leqslant k} f\left(D_{i_{1}} \cap \ldots \cap D_{i_{p}}\right)
\end{gathered}
$$

We show that all terms in the sum (5) represent integers. By the intersection stability of $\Omega$ and the form (4) of the sets $D_{j}$ we see that $T=D_{i_{1}} \cap \ldots \cap D_{i_{p}}$ takes a form corresponding to $(4)$.

$$
T=A_{i_{0}} \cap \bar{A}_{i_{1}} \cap \ldots \cap \bar{A}_{i_{r}}
$$

Every set $A_{i_{j}}$ that occurs in (6) belongs to $\Omega$ with the possible exception $A_{i_{0}}=M$. Now we conclude by Lemma 2 that $f(T)$ is integral.

\section{Group characters and Cayley graphs}

Lovász [12] (see also Babai [3]) developed a method to express the eigenvalues of a graph in terms of the characters of a transitive subgroup of its automorphism group. This theory simplifies considerably for Cayley graphs over abelian groups. To improve the readability of our paper we include Lovász's arguments reduced to our purposes (Lemma 4, 6, and 7). For more algebraic background we refer to Cohn [6].

Let $\Gamma$ be a finite additive group with $n$ elements, $|\Gamma|=n$. For a positive integer $k$ and $a \in \Gamma$ we denote as usual by $k a$ the $k$-fold sum of $a$ to itself, $(-k) a=k(-a), 0 a=0$. A character $\psi$ of $\Gamma$ is a homomorphism from $\Gamma$ into the multiplicative group of complex numbers, $\psi: \Gamma \rightarrow \mathbb{C} \backslash\{0\}$,

$$
\psi(\mu a+\nu b)=(\psi(a))^{\mu}(\psi(b))^{\nu} \text { for every } a, b \in \Gamma \text { and } \mu, \nu \in Z \text {. }
$$

Fermat's little theorem yields

$$
(\psi(a))^{n}=\psi(n a)=\psi(0)=1 .
$$

Therefore, $\psi(a)$ is an $n$-th root of unity for every $a \in \Gamma$.

Lemma 4. Let $H$ be a subgroup of $\Gamma$ and $\psi$ a character of $\Gamma$. If $H$ contains an element $g$ with $\psi(g) \neq 1$, then $\psi(H)=0$ else $\psi(H)=|H|$.

Proof. If $g \in H$ and $\psi(g) \neq 1$ then we have

$$
\psi(H)=\sum_{h \in H} \psi(h+g)=\psi(g) \psi(H),(1-\psi(g)) \psi(H)=0
$$

which implies $\psi(H)=0$. If $\psi(g)=1$ for every $g \in H$ then $\psi(H)=|H|$. 
We denote by $B(\Gamma)$ the Boolean algebra generated by the subgroups of $\Gamma$.

Lemma 5. For an arbitrary character $\psi$ of $\Gamma$ every set $S \in B(\Gamma)$ is $\psi$-integral.

Proof. According to Lemma 4 every subgroup $H$ of $\Gamma$ is $\psi$-integral. The subgroups of $\Gamma$ constitute an intersection stable set system including $\Gamma$ itself. Lemma 3 implies that every set $S \in B(\Gamma)$ is $\psi$-integral.

Lemma 6. Let $\psi$ be a character of the additive group $\Gamma=\left\{v_{1}, \ldots, v_{n}\right\}, S \subseteq \Gamma, 0 \notin$ $S,-S=S$. Assume that $A=\left(a_{i, j}\right)$ is the adjacency matrix of $G=C a y(\Gamma, S)$ with respect to the given ordering of the vertex set $V(G)=\Gamma$. Then the column vector $\left(\psi\left(v_{j}\right)\right)_{j=1, \ldots, n}$ is an eigenvector of $A$ with eigenvalue $\psi(S)$.

Proof. We evaluate the product of the $i$-th row of $A$ and $\left(\psi\left(v_{j}\right)\right)_{j=1, \ldots, n}$.

$$
\begin{aligned}
\sum_{j=1}^{n} a_{i, j} \psi\left(v_{j}\right) & =\sum_{1 \leqslant j \leqslant n, v_{j}-v_{i} \in S} \psi\left(v_{j}\right)=\sum_{s \in S} \psi\left(s+v_{i}\right) \\
& =\psi\left(v_{i}\right) \sum_{s \in S} \psi(s)=\psi\left(v_{i}\right) \psi(S)
\end{aligned}
$$

From now on we assume that the finite additive group $\Gamma$ is abelian. Then $\Gamma$ can be represented as the direct product of cyclic groups of prime power order (see Cohn [6]).

$$
\Gamma=Z_{n_{1}} \otimes \cdots \otimes Z_{n_{k}},|\Gamma|=n=n_{1} \cdots n_{k}
$$

We consider the elements $x \in \Gamma$ as elements of the cartesian product $Z_{n_{1}} \times \cdots \times Z_{n_{k}}$,

$$
x=\left(x_{i}\right), x_{i} \in Z_{n_{i}}=\left\{0,1, \ldots, n_{i}-1\right\}, 1 \leqslant i \leqslant k .
$$

Addition is coordinatewise modulo $n_{i}$. Denote by $e_{i}$ the unit vector with entry 1 in position $i$ and entry 0 in all positions $j \neq i$. A character $\psi$ of $\Gamma$ is uniquely determined by its values $\psi\left(e_{i}\right), 1 \leqslant i \leqslant k$.

$$
x=\left(x_{i}\right)=\sum_{i=1}^{k} x_{i} e_{i}, \quad \psi(x)=\prod_{i=1}^{k}\left(\psi\left(e_{i}\right)\right)^{x_{i}}
$$

As $e_{i} \in \Gamma$ has order $n_{i}$, the value $\psi\left(e_{i}\right)$ must be a complex $n_{i}$-th root of unity. So there are $n_{i}$ possible choices for the value of $\psi\left(e_{i}\right)$. Let $\zeta_{i}$ be a primitive $n_{i}$-th root of unity for every $i, 1 \leqslant i \leqslant k$. For every $\alpha=\left(\alpha_{i}\right) \in \Gamma$ a character $\psi_{\alpha}$ can be uniquely defined by

$$
\psi_{\alpha}\left(e_{i}\right)=\zeta_{i}^{\alpha_{i}}, 1 \leqslant i \leqslant k .
$$

Thus all $|\Gamma|=n$ characters of the abelian group $\Gamma$ can be obtained. 
Lemma 7. Let $\psi_{1}, \ldots, \psi_{n}$ be the distinct characters of the additive abelian group $\Gamma=$ $\left\{v_{1}, \ldots, v_{n}\right\}, S \subseteq \Gamma, 0 \notin S,-S=S$. Assume that $A=\left(a_{i, j}\right)$ is the adjacency matrix of $G=\operatorname{Cay}(\Gamma, S)$ with respect to the given ordering of the vertex set $V(G)=\Gamma$. Then the column vectors $\left(\psi_{i}\left(v_{j}\right)\right)_{j=1, \ldots, n}, 1 \leqslant i \leqslant n$, constitute an orthogonal basis of $\mathbb{C}^{n}$ consisting of eigenvectors of $A$. To the eigenvector $\left(\psi_{i}\left(v_{j}\right)\right)_{j=1, \ldots, n}$ belongs the eigenvalue $\psi_{i}(S)$.

Proof. By Lemma 6 and the considerations above it remains to prove that for $\alpha=\left(\alpha_{i}\right) \in$ $\Gamma, \beta=\left(\beta_{i}\right) \in \Gamma, \alpha \neq \beta$, the eigenvectors $\left(\psi_{\alpha}\left(v_{j}\right)\right)_{j=1, \ldots, n}$ and $\left(\psi_{\beta}\left(v_{j}\right)\right)_{j=1, \ldots, n}$ are orthogonal (with respect to the standard inner product of $\mathbb{C}^{n}$ ). We represent $\Gamma$ by $(7)$ and define $\psi_{\alpha}$ and $\psi_{\beta}$ according to (8) and (9). Observe that the complex conjugate $\bar{\zeta}$ of a root of unity $\zeta$ satisfies $\bar{\zeta}=\zeta^{-1}$.

$$
\begin{aligned}
\sigma & =\sum_{j=1}^{n} \psi_{\alpha}\left(v_{j}\right) \overline{\psi_{\beta}\left(v_{j}\right)}=\sum_{x=\left(x_{i}\right) \in \Gamma} \prod_{i=1}^{k}\left(\zeta_{i}\right)^{\alpha_{i} x_{i}} \prod_{i=1}^{k}\left(\bar{\zeta}_{i}\right)^{\beta_{i} x_{i}} \\
& =\sum_{0 \leqslant x_{1}<n_{1}} \ldots \sum_{0 \leqslant x_{k}<n_{k}} \prod_{i=1}^{k} \zeta_{i}^{\left(\alpha_{i}-\beta_{i}\right) x_{i}}=\prod_{i=1}^{k} \sum_{0 \leqslant x_{i}<n_{i}} \zeta_{i}^{\left(\alpha_{i}-\beta_{i}\right) x_{i}}
\end{aligned}
$$

As $\alpha \neq \beta$ we may assume e.g. $\alpha_{1} \neq \beta_{1}$. Then

$$
\sum_{0 \leqslant x_{1}<n_{1}} \zeta_{1}^{\left(\alpha_{1}-\beta_{1}\right) x_{1}}=\frac{\zeta_{1}^{\left(\alpha_{1}-\beta_{1}\right) n_{1}}-1}{\zeta_{1}^{\left(\alpha_{1}-\beta_{1}\right)}-1}=0
$$

implies $\sigma=0$ by (10).

Our main result is stated in the next theorem.

Theorem 8. Let $\Gamma$ be a finite abelian group and $B(\Gamma)$ the Boolean algebra generated by the subgroups of $\Gamma$. For every set $S \in B(\Gamma), 0 \notin S$, the Cayley graph Cay $(\Gamma, S)$ is integral.

Proof. According to Lemma 7 all eigenvalues of $\operatorname{Cay}(\Gamma, S)$ have the form $\psi(S)$ with a character $\psi$ of $\Gamma$. By Lemma 5 we know that $\psi(S)$ is integral for every $S \in B(\Gamma)$.

For an integer $n \geqslant 2$ and a proper divisor $d$ of $n$ we define

$$
G_{n}(d)=\left\{k \in Z_{n}: \operatorname{gcd}(k, n)=d\right\} .
$$

The following result of So [15] leads to the converse of Theorem 8 for cyclic groups.

Lemma 9. Let $n$ be an integer, $n \geqslant 2, S \subseteq Z_{n}, 0 \notin S,-S=S$. The Cayley graph $\operatorname{Cay}\left(Z_{n}, S\right)$ is integral, if and only if there are proper divisors $d_{1}, \ldots, d_{r}$ of $n$ such that

$$
S=\bigcup_{j=1}^{r} G_{n}\left(d_{j}\right)
$$


Theorem 10. Let $n$ be an integer, $n \geqslant 2, S \subseteq Z_{n}, 0 \notin S,-S=S$. The Cayley graph $\operatorname{Cay}\left(Z_{n}, S\right)$ is integral, if and only if $S \in B\left(Z_{n}\right)$.

Proof. If $S \in B\left(Z_{n}\right)$ then $\operatorname{Cay}\left(Z_{n}, S\right)$ is integral by Theorem 8 . To prove the converse let $\operatorname{Cay}\left(Z_{n}, S\right)$ be integral. By Lemma 9 there are proper divisors $d_{1}, \ldots, d_{r}$ of $n$ such that $S$ satisfies (11). To prove $S \in B\left(Z_{n}\right)$ it is sufficient to show that $G_{n}(d) \in B\left(Z_{n}\right)$ for every proper divisor $d$ of $n$. To every proper divisor $d$ of $n$ the cyclic group $Z_{n}$ has exactly one subgroup of order $(n / d)>1$, namely the cyclic group $[d]$ generated by $d$. If we define

$$
M_{n}(d)=[d] \backslash\{0\}=\left\{q d: 1 \leqslant q<\frac{n}{d}\right\}
$$

then $M_{n}(d) \in B\left(Z_{n}\right)$. Now we obtain

$$
\begin{aligned}
G_{n}(d) & =\left\{q d: 1 \leqslant q<\frac{n}{d}, \operatorname{gcd}\left(q, \frac{n}{d}\right)=1\right\} \\
& =M_{n}(d) \backslash \bigcup\left\{M_{n}(\delta d): 1<\delta<\frac{n}{d}, \delta \text { divides } \frac{n}{d}\right\},
\end{aligned}
$$

which implies $G_{n}(d) \in B\left(Z_{n}\right)$.

In the introductory section we defined a finite additive group $\Gamma$ to be Cayley integral, if for every $S \subseteq \Gamma, 0 \notin S,-S=S$, the Cayley graph $\operatorname{Cay}(\Gamma, S)$ is integral. Observe that for this definition $\Gamma$ may be nonabelian. By $\operatorname{ord}(a)$ we denote the order of $a \in \Gamma$.

Lemma 11. If the finite group $\Gamma$ is Cayley integral then

$$
\operatorname{ord}(a) \in\{2,3,4,6\} \text { for every } a \in \Gamma, a \neq 0 .
$$

Proof. The eigenvalues of a circuit $C_{n}$ of length $n \geqslant 3$ are (see [5])

$$
\lambda_{j}=2 \cos \left(\frac{2 \pi}{n} j\right), j=0,1, \ldots, n-1 .
$$

This implies that $C_{n}$ is integral only for $n=3,4$, or 6 . Assume that $\Gamma$ is Cayley integral and contains an element $a \neq 0$, ord $(a) \notin\{2,3,4,6\}$. Let $U=[a]$ and $S=\{a,-a\}$. Then $|S|=2$ and the subgroup generated by $S$ is $[S]=U$. The Cayley graph $G=\operatorname{Cay}(\Gamma, S)$ is regular of degree $|S|=2$. Its connected components are generated by the right cosets of $U$. They are circuits of length $|U|=\operatorname{ord}(a) \notin\{3,4,6\}$. Therefore, $G$ is not integral, contradicting our assumption on $\Gamma$.

Lemma 12. Let $\Gamma$ be a finite additive group, $S \subseteq \Gamma,-S=S$. If $\operatorname{ord}(a) \in\{2,3,4,6\}$ for every $a \in S$ then $S \in B(\Gamma)$.

Proof. We show $\{a,-a\} \in B(\Gamma)$ for every $a \in S$. This leads to

$$
S=\bigcup_{a \in S}\{a,-a\} \in B(\Gamma) .
$$

According to the four possible orders of $a \in S$ we consider four cases.

1) $\operatorname{ord}(a)=2:\{a,-a\}=\{a\}=[a] \backslash\{0\} \in B(\Gamma)$.

2) $\operatorname{ord}(a)=3:\{a,-a\}=[a] \backslash\{0\} \in B(\Gamma)$.

3) $\operatorname{ord}(a)=4:\{a,-a\}=[a] \backslash\{0,2 a\}=[a] \backslash[2 a] \in B(\Gamma)$.

4) $\operatorname{ord}(a)=6:\{a,-a\}=[a] \backslash\{0,2 a, 3 a, 4 a\}=[a] \backslash([2 a] \cup[3 a]) \in B(\Gamma)$. 
Denote by $Z_{k}^{n}$ the $n$-fold direct product of $Z_{k}$ with itself. It was already noticed by Lovász [12] that all Cayley graphs over $Z_{2}^{n}$ ("cubelike graphs") are integral. The following theorem extends this result.

Theorem 13. All nontrivial abelian Cayley integral groups are represented by

$$
Z_{2}^{n}, Z_{3}^{n}, Z_{4}^{n}, Z_{2}^{m} \otimes Z_{3}^{n}, Z_{2}^{m} \otimes Z_{4}^{n}, m \geqslant 1, n \geqslant 1 .
$$

Proof. Lemma 11, Lemma 12, and Theorem 8 imply that the abelian group $\Gamma$ is Cayley integral, if and only if

$$
\operatorname{ord}(a) \in\{2,3,4,6\} \text { for every } a \in \Gamma, a \neq 0 .
$$

The abelian group $\Gamma$ is the direct product of cyclic groups of prime power order. In (12) we have listed all types of nontrivial abelian groups which satisfy (13).

\section{Examples}

\subsection{Hamming graphs}

Let $m_{1}, \ldots, m_{r}$ be positive integers, $D=\left\{d_{1}, \ldots d_{k}\right\}$ a set of integers $d_{i}, 1 \leqslant d_{i} \leqslant r$. The Hamming graph $H=\operatorname{Ham}\left(m_{1}, \ldots, m_{r} ; D\right)$ has as its vertex set the abelian group

$$
\Gamma=Z_{m_{1}} \otimes \cdots \otimes Z_{m_{r}} .
$$

The Hamming distance of vertices $x=\left(x_{i}\right) \in \Gamma$ and $y=\left(y_{i}\right) \in \Gamma$ is

$$
d(x, y)=\left|\left\{i: 1 \leqslant i \leqslant r, x_{i} \neq y_{i}\right\}\right| .
$$

Vertices $x$ and $y$ are adjacent in $H$, if $d(x, y) \in D$. We show $H=C a y(\Gamma, S)$ with $S \in B(\Gamma)$. Then Theorem 8 implies that $H$ is integral.

The weight of $x=\left(x_{i}\right) \in \Gamma$ is

$$
w(x)=\left|\left\{i: 1 \leqslant i \leqslant r, x_{i} \neq 0\right\}\right| .
$$

We achieve $H=\operatorname{Cay}(\Gamma, S)$ by

$$
S=S_{1} \cup \ldots \cup S_{k}, \quad S_{j}=\left\{x \in \Gamma: w(x)=d_{j}\right\} \text { for } 1 \leqslant j \leqslant k .
$$

It remains to show $S_{j} \in B(\Gamma)$ for every $j, 1 \leqslant j \leqslant k$, or generally

$$
S(d)=\{x \in \Gamma: w(x)=d\} \in B(\Gamma) \text { for every } d, 1 \leqslant d \leqslant r .
$$

Define the support of $x=\left(x_{i}\right) \in \Gamma$ by $\operatorname{supp}(x)=\left\{i: 1 \leqslant i \leqslant r, x_{i} \neq 0\right\}$.

Let $\left\{A_{q}: 1 \leqslant q \leqslant\left(\begin{array}{l}r \\ d\end{array}\right)\right\}$ be the family of all $d$-element subsets of $\{1, \ldots, r\}$ and

$$
B_{q}=\left\{x \in S(d): \operatorname{supp}(x)=A_{q}\right\} \text { for } 1 \leqslant q \leqslant\left(\begin{array}{l}
r \\
d
\end{array}\right) .
$$


Then we have

$$
S(d)=\bigcup_{1 \leqslant q \leqslant\left(\begin{array}{l}
r \\
d
\end{array}\right)} B_{q} .
$$

So it is sufficient to show $B_{q} \in B(\Gamma)$ for every $q, 1 \leqslant q \leqslant\left(\begin{array}{l}r \\ d\end{array}\right)$.

If for $A \subseteq\{1, \ldots, r\}$ we define

$$
P(A)=\left\{x=\left(x_{i}\right) \in \Gamma: x_{i}=0 \text { for every } i \notin A\right\}
$$

then $P(A)$ is a subgroup of $\Gamma$, which by (14) is isomorphic to $\bigotimes_{i \in A} Z_{m_{i}}$. By (15) we now conclude

$$
B_{q}=P\left(A_{q}\right) \backslash \bigcup_{\substack{\subset \\ A_{f q}}} P(A) \in B(\Gamma) .
$$

Thus we arrive at the following result.

Proposition 14. Every Hamming graph $\operatorname{Ham}\left(m_{1}, \ldots, m_{r} ; D\right)$ is an integral Cayley graph.

\subsection{Sudoku graphs}

For an integer $n \geqslant 2$ an $n$-Sudoku is an arrangement of $n \times n$ square blocks each consisting of $n \times n$ cells. In Figure 1 we display an example for the commonly used format given by $n=3$. Each cell has to be filled with a number (color) ranging from 1 to $n^{2}$ such that every block, row or column contains all of the colors $1, \ldots, n^{2}$. For a Sudoku puzzle certain colored cells are stipulated (in Figure 1 in bold type). The aim is to color the remaining cells according to the above conditions. This puzzle may be considered as the task to complete a partial proper coloring of the underlying graph $\operatorname{Sud}(n)$ to a proper coloring of this graph.

\begin{tabular}{||l|l|l||l|l|l||l|l|l||}
\hline \hline $\mathbf{9}$ & 1 & 4 & 2 & 5 & 6 & $\mathbf{3}$ & 7 & $\mathbf{8}$ \\
\hline 7 & 6 & 5 & $\mathbf{3}$ & 1 & $\mathbf{8}$ & 2 & 9 & 4 \\
\hline 3 & 8 & 2 & 7 & $\mathbf{9}$ & 4 & $\mathbf{6}$ & 5 & $\mathbf{1}$ \\
\hline \hline 1 & $\mathbf{2}$ & 6 & 9 & 8 & $\mathbf{7}$ & 5 & $\mathbf{4}$ & 3 \\
\hline 5 & 4 & $\mathbf{7}$ & 6 & 3 & $\mathbf{2}$ & $\mathbf{1}$ & 8 & $\mathbf{9}$ \\
\hline 8 & $\mathbf{9}$ & 3 & $\mathbf{1}$ & $\mathbf{4}$ & 5 & 7 & $\mathbf{6}$ & 2 \\
\hline \hline $\mathbf{6}$ & 5 & $\mathbf{1}$ & 8 & $\mathbf{2}$ & 9 & $\mathbf{4}$ & 3 & 7 \\
\hline 4 & 3 & 9 & $\mathbf{5}$ & 7 & $\mathbf{1}$ & 8 & 2 & 6 \\
\hline $\mathbf{2}$ & 7 & $\mathbf{8}$ & 4 & $\mathbf{6}$ & 3 & 9 & 1 & $\mathbf{5}$ \\
\hline \hline
\end{tabular}

Figure 1

The $S u d o k u$ graph $S u d(n)$ has as its vertices the $n^{2}$ cells of an $n$-Sudoku. Vertices (cells) are adjacent, if they are in the same block, row or column. Based on the representation of $\operatorname{Sud}(n)$ as a certain product (NEPS) of complete graphs it has been shown in [13] that $\operatorname{Sud}(n)$ is integral. Its eigenvalues (multiplicities in brackets) in descending order are:

$$
\begin{aligned}
& 3 n^{2}-2 n-1[1], 2 n^{2}-2 n-1[2(n-1)], n^{2}-n-1[2 n(n-1)], \\
& n^{2}-2 n-1\left[(n-1)^{2}\right],-1\left[n^{2}(n-1)^{2}\right],-1-n\left[2 n(n-1)^{2}\right] .
\end{aligned}
$$


Here we show $\operatorname{Sud}(n)=\operatorname{Cay}(\Gamma, S)$ for an abelian group $\Gamma$ and $S \in B(\Gamma)$. So $S u d(n)$ is an integral Cayley graph according to Theorem 8 . The above eigenvalues could also be determined by Lovász's method, Lemma 7.

We represent the vertices (cells) of $\operatorname{Sud}(n)$ by the elements

$$
x=\left(x_{1}, x_{2}, x_{3}, x_{4}\right) \in \Gamma=Z_{n}^{4}, \quad Z_{n}=\{0,1, \ldots, n-1\} .
$$

For a given cell the first pair $\left(x_{1}, x_{2}\right)$ of coordinates localizes the block of the cell. The second pair $\left(x_{3}, x_{4}\right)$ describes the position of the cell within its block. According to the different types of edges in $\operatorname{Sud}(n)$ the set $S$ is partitioned into three subsets, $S=S_{1} \cup S_{2} \cup S_{3}$,

$$
\begin{aligned}
& S_{1}=\left\{\left(0,0, x_{3}, x_{4}\right): x_{3}, x_{4} \in Z_{n},\left(x_{3}, x_{4}\right) \neq(0,0)\right\} \\
& S_{2}=\left\{\left(0, x_{2}, 0, x_{4}\right): x_{2}, x_{4} \in Z_{n}, x_{2} \neq 0\right\} \\
& S_{3}=\left\{\left(x_{1}, 0, x_{3}, 0\right): x_{1}, x_{3} \in Z_{n}, x_{1} \neq 0\right\} .
\end{aligned}
$$

Edges within a block are provided by $S_{1}$. The remaining edges within a row or within a column are provided by $S_{2}$ and $S_{3}$. Thus we achieve $\operatorname{Sud}(n)=\operatorname{Cay}(\Gamma, S)$. Let $Z_{1}=\{0\}$.

$$
\begin{aligned}
& S_{1}=Z_{1} \otimes Z_{1} \otimes Z_{n} \otimes Z_{n} \backslash Z_{1} \otimes Z_{1} \otimes Z_{1} \otimes Z_{1} \text { implies } S_{1} \in B(\Gamma) . \\
& S_{2}=Z_{1} \otimes Z_{n} \otimes Z_{1} \otimes Z_{n} \backslash Z_{1} \otimes Z_{1} \otimes Z_{1} \otimes Z_{n} \text { implies } S_{2} \in B(\Gamma) . \\
& S_{3}=Z_{n} \otimes Z_{1} \otimes Z_{n} \otimes Z_{1} \backslash Z_{1} \otimes Z_{1} \otimes Z_{n} \otimes Z_{1} \text { implies } S_{3} \in B(\Gamma) .
\end{aligned}
$$

Therefore, $S \in B(\Gamma)$ and $\operatorname{Sud}(n)=\operatorname{Cay}(\Gamma, S)$ is an integral Cayley graph by Theorem 8 .

In a variant of Sudoku, positional Sudoku, discussed by Elsholtz and Mütze [7] the cells have to satisfy an additional condition. Distinct cells in the same position of their respective blocks have to be colored differently. The underlying positional Sudoku graph $\operatorname{SudP}(n)$ gets additional edges in comparison to $\operatorname{Sud}(n)$. In the Cayley graph representation these edges are established by $S_{4}=\left\{\left(x_{1}, x_{2}, 0,0\right): x_{1}, x_{2} \in Z_{n}, x_{1} \neq 0, x_{2} \neq 0\right\}$.

$$
S_{4}=Z_{n} \otimes Z_{n} \otimes Z_{1} \otimes Z_{1} \backslash\left(Z_{1} \otimes Z_{n} \otimes Z_{1} \otimes Z_{1} \cup Z_{n} \otimes Z_{1} \otimes Z_{1} \otimes Z_{1}\right) .
$$

This implies $S_{4} \in B(\Gamma)$ and $\tilde{S}=S_{1} \cup S_{2} \cup S_{3} \cup S_{4} \in B(\Gamma)$. Therefore, $\operatorname{SudP}(n)=\operatorname{Cay}(\Gamma, \tilde{S})$ is an integral Cayley graph by Theorem 8. Its eigenvalues can be determined by Lovász's method, Lemma 7. We list them in descending order (multiplicities in brackets).

$$
\begin{aligned}
& 4 n(n-1)[1], 2 n^{2}-3 n[4(n-1)], n(n-2)\left[4(n-1)^{2}\right], \\
& 0\left[(n-1)^{4}\right],-n\left[4(n-1)^{3}\right]-2 n\left[2(n-1)^{2}\right]
\end{aligned}
$$

We summarize the main results of this subsection.

Proposition 15. Every Sudoku graph $S u d(n)$ and every positional Sudoku graph $S u d P(n)$ is an integral Cayley graph. 


\subsection{Pandiagonal Latin square graphs}

A Latin square is an $n \times n$-matrix with entries from $\{1, \ldots, n\}$ such that every number $1, \ldots, n$ appears exactly once in every row and in every column. For a pandiagonal Latin square two additional conditions have to be satisfied. Every number $1, \ldots, n$ has to appear exactly once in the main diagonal and its broken parallels as well as in the secondary diagonal and its broken parallels. Hedayat [10] proved that an $n \times n$-pandiagonal Latin square exists, if and only if $n \equiv \pm 1$ modulo 6 . Figure 2 presents a $7 \times 7$-pandiagonal Latin square.

\begin{tabular}{|l|l|l|l|l|l|l|}
\hline 1 & 2 & 3 & 4 & 5 & 6 & 7 \\
\hline 6 & 7 & 1 & 2 & 3 & 4 & 5 \\
\hline 4 & 5 & 6 & 7 & 1 & 2 & 3 \\
\hline 2 & 3 & 4 & 5 & 6 & 7 & 1 \\
\hline 7 & 1 & 2 & 3 & 4 & 5 & 6 \\
\hline 5 & 6 & 7 & 1 & 2 & 3 & 4 \\
\hline 3 & 4 & 5 & 6 & 7 & 1 & 2 \\
\hline
\end{tabular}

Figure 2

For $n \geqslant 2$ the pandiagonal Latin square graph $P L S G(n)$ has as its vertex set the $n^{2}$ positions of an $n \times n$-matrix. Distinct vertices (positions) are adjacent, if they are in the same row, in the same column, in the same (broken) parallel to the main diagonal or in the same (broken) parallel to the secondary diagonal. The graph $P L S G(n)$ is defined for every integer $n \geqslant 2$. The existence of an $n \times n$-pandiagonal Latin square is equivalent to chromatic number $\chi(P L S G(n))=n$. Here we show that $P L S G(n)=\operatorname{Cay}(\Gamma, S)$ is an integral Cayley graph.

Naturally, we describe the vertex set of $P L S G(n)$ by $\Gamma=Z_{n} \otimes Z_{n}$. The set $S$ is partitioned into four parts, $S=S_{1} \cup S_{2} \cup S_{3} \cup S_{4}$, according to the four types of edges in $P L S G(n)$. The sets

$$
S_{1}=\left\{\left(0, x_{2}\right): x_{2} \in Z_{n}, x_{2} \neq 0\right\}, \quad S_{2}=\left\{\left(x_{1}, 0\right): x_{1} \in Z_{n}, x_{1} \neq 0\right\}
$$

provide the edges between positions in the same row or column. With the notation $Z_{1}=\{0\}$ we see

$$
S_{1}=Z_{1} \otimes Z_{n} \backslash Z_{1} \otimes Z_{1} \in B(\Gamma), \quad S_{2}=Z_{n} \otimes Z_{1} \backslash Z_{1} \otimes Z_{1} \in B(\Gamma) .
$$

The set $S_{3}=\left\{\left(x_{1}, x_{1}\right): x_{1} \in Z_{n}, x_{1} \neq 0\right\}$ provides the edges between positions in the main diagonal or in one of its broken parallels. As $U_{1}=\left\{\left(x_{1}, x_{1}\right): x_{1} \in Z_{n}\right\}$ is a subgroup of $\Gamma$, we conclude $S_{3}=U_{1} \backslash Z_{1} \otimes Z_{1} \in B(\Gamma)$. The set

$$
S_{4}=\left\{\left(x_{1},-x_{1}\right): x_{1} \in Z_{n}, x_{1} \neq 0, x_{1} \neq \frac{n}{2} \text { for even } n\right\}
$$

provides the remaining edges between positions in the secondary diagonal or one of its broken parallels. If we define

$U_{2}=\left\{\left(x_{1},-x_{1}\right): x_{1} \in Z_{n}\right\}, U_{3}=\left\{(0,0),\left(\frac{n}{2}, \frac{n}{2}\right)\right\}$ for even $n$ and $U_{3}=\{(0,0)\}$ for odd $n$, 
then $U_{2}$ and $U_{3}$ are subgroups of $\Gamma$ and $S_{4}=U_{2} \backslash U_{3} \in B(\Gamma)$. Now we arrive at $P L S G(n)=$ $\operatorname{Cay}(\Gamma, S), S=S_{1} \cup S_{2} \cup S_{3} \cup S_{4} \in B(\Gamma)$. Theorem 8 implies that $P L S G(n)$ is an integral Cayley graph.

Proposition 16. Every pandiagonal Latin square graph $P L S G(n)$ is an integral Cayley graph.

We have determined the eigenvalues of PLSG(n) by Lovász's method, Lemma 7 . The fact that there are exactly three different eigenvalues for odd $n \geqslant 5$ reflects that $P L S G(n)$ is strongly regular in this case (see [11]). We list the eigenvalues in descending order (multiplicities in brackets).

$$
\begin{array}{ll}
\text { Case } n \text { odd: } & 4 n-4[1], n-4[4 n-4],-4\left[n^{2}-4 n+3\right] . \\
\text { Case } n \text { even: } & 4 n-5[1], 2 n-5[1], n-3[n], \\
& n-5[3 n-6],-3\left[\frac{n^{2}}{2}-n\right],-5\left[\frac{n^{2}}{2}-3 n+4\right] .
\end{array}
$$

\section{$5 \quad$ Problems and remarks}

1. We think that the converse of Theorem 8 is not only true for cyclic groups (Theorem 10), but for abelian groups in general. By a computer search we found no counterexample to this conjecture among abelian groups up to order 71 .

2. There are nonabelian groups, e.g. all dihedral groups $D_{n},\left|D_{n}\right|=2 n \geqslant 8$, for which Theorem 8 does not hold.

3. Up to order 12 we have found three nonabelian Cayley integral groups: the symmetric group $S_{3}$ of order 6 , the group $Q_{8}$ of quaternions as a group of order 8, and the semidirect product (see Cohn [6]) of $Z_{3}$ and $Z_{4}$ as a group of order 12. Determine all nonabelian Cayley integral groups.

4. Let the finite abelian group $\Gamma$ be represented as the direct product of cyclic groups, $\Gamma=Z_{m_{1}} \otimes \cdots \otimes Z_{m_{r}}$. Describe an effective method to decide for a subset $S \subseteq \Gamma$, if $S$ belongs to the Boolean algebra $B(\Gamma)$ generated by the subgroups of $\Gamma$.

5. From the existence theorem of Hedayat we know that $\chi(P L S G(n))=n$, if $n \equiv \pm 1$ modulo 6. Determine the chromatic number $\chi(P L S G(n))$ in the remaining cases.

\section{References}

[1] Abdollahi, A., And Vatandoost, E. Which Cayley graphs are integral? Electronic J. Comb. 16(1) (2009), R122, 1-17.

[2] Ahmadi, O., Alon, N., Blake, L. F., and Shparlinski, I. E. Graphs with integral spectrum. Linear Alg. Appl. 430 (2009), 547-552. 
[3] Babai, L. Spectra of Cayley graphs. J. Combinatorial Theory B 27 (1979), 180-189.

[4] Balinska, K., Cvetković, D., Rodosavluević, Z., Simić, S., and Stevanović, D. A survey on integral graphs. Univ. Beograd, Publ. Elektrotehn. Fak. Ser. Mat 13 (2003), 42-65.

[5] Biggs, N. Algebraic graph theory. Second Edition. Cambridge Mathematical Library. Cambridge University Press, 1993.

[6] Cohn, P. M. Basic Algebra. Springer, London, 2003.

[7] Elsholtz, C., And Mütze, A. Sudoku im Mathematikunterricht. Math. Semesterber. 39 (2007), 69-93.

[8] Godsil, C., And Royle, G. Algebraic graph theory. Graduate Texts in Mathematics. Vol 207. Springer, 2001.

[9] Harary, F., And Schwenk, A. J. Which graphs have integral spectra? Lecture Notes in Mathematics 406, Springer Verlag (1974), 45-50.

[10] Hedayat, A. A complete solution to the existence and nonexistence of Knut Vik designs and orthogonal Knut Vik designs. J. Combinatorial Theory (A) 22 (1977), 331-337.

[11] van Lint, J. H., And Wilson, R. M. A course in combinatorics. Cambridge University Press, 1992.

[12] LovÁsz, L. Spectra of graphs with transitive groups. Priodica Mathematica Hungarica 6 (1975), 191-195.

[13] SAnder, T. Sudoku graphs are integral. Electronic J. Combinatorics 16(1) (2009), N25, 1-7.

[14] Sander, T. Eigenspaces of Hamming graphs and unitary Cayley graphs. Ars Mathematica Contemporanea, to appear.

[15] So, W. Integral circulant graphs. Discrete Mathematics 306 (2005), 153-158. 